\title{
Methodological considerations regarding cognitive interventions in dementia
}

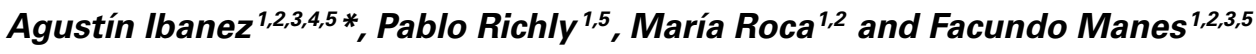 \\ ${ }^{1}$ Laboratory of Experimental Psychology and Neuroscience (LPEN), Institute of Cognitive Neurology (INECO), Favaloro University, Buenos Aires, Argentina \\ 2 UDP-INECO Foundation Core on Neuroscience (UIFCoN), Diego Portales University, Santiago, Chile \\ ${ }^{3}$ National Scientific and Technical Research Council (CONICET), Buenos Aires, Argentina \\ ${ }^{4}$ Universidad Autónoma del Caribe, Barranquilla, Colombia \\ ${ }^{5}$ Australian Research Council Centre of Excellence in Cognition and its Disorders, Sydney, NSW, Australia \\ *Correspondence: aibanez@ineco.org.ar \\ Edited by: \\ P. Hemachandra Reddy, Oregon Health and Science University, USA \\ Reviewed by: \\ P. Hemachandra Reddy, Oregon Health and Science University, USA \\ Russell H. Swerdlow, University of Kansas Medical Center, USA
}

Keywords: cognitive intervention, dementia, methods development, pre/post measurement, cognition, neuropsychology, neuroscience

Dementia causes massive cognitive, affective, and social impairment as well as concomitant functional decline. Cognitive interventions, together with pharmacological treatments, are acknowledged as important tools to delay mental weakening in dementing populations and to preserve the life quality of patients and their relatives (Prince et al., 2011; Woods et al., 2012). Given the socioeconomic impact of dementia on the health system, it is critical to assess cognitive intervention techniques in terms of cost-efficiency (Hurd et al., 2013). Nevertheless, most recent reports have neglected this issue, showing small, non-replicated, or even null results (Olazarán et al., 2010). Arguably, this is partly so because researchers are more specialized in the study of impairments than in the design of intervention programs. Consequently, there is no agreement on how to define cognitive intervention or how to measure its success (Giordano et al., 2010; Fernández-Prado et al., 2012).

A problem implicitly evident in relevant meta-analyses is that methodological decisions affect the results' reliability and replication (Bahar-Fuchs et al., 2013a). Usually, an intervention program involves participant inclusion criteria, pre-intervention measurements, training sessions, postintervention measurements, and pre-post statistical analyses to unravel the possible intervention effects. However, systematic methodological considerations for cognitive intervention in dementia are scant (Wollen, 2010). To increase the efficiency of cognitive stimulation assessment, this paper sets forth guidelines for cognitive intervention design, control group formation, control condition manipulation, pre/post-intervention measurement, and statistical analysis (see Table 1).

\section{PARTICIPANTS}

Participant selection is a critical issue, which affects study design. First, the sample size should be sufficiently large (approximately $>100$ ). This allows to control for possible confounders and to prevent the sample from becoming too small during follow-up. However, this is often not easy to achieve, given the difficulty to recruit patients with similar clinical characteristics (e.g., dementia subtype, pharmacological treatment and doses, disease duration and severity, and caregiver support). Current reports usually consider really modest samples (Niu et al., 2010; Dröes et al., 2011; Viola et al., 2011).

Different approaches should be considered for small and large groups. In small groups, intervention should be focused and specific (e.g., restricted to one cognitive domain) and based on relevant pre/post measures. For their own part, larger samples allow to track and control for confounding variables. Although a full clinical characterization is always desirable, the impact of these variables can be tracked only with large sample sizes.

Moreover, depending on the dementia subtype, diagnosis can be probable [e.g., behavioral variant of frontotemporal dementia (bvFTD)] or confirmed (e.g., Huntington's disease with confirmed mutation). For probable diagnosis, more clinical/neuropsychological control measures should be considered. Moreover, intervention effects can be subtle and difficult to identify in conditions with rapid progression. Thus, different control and intervention strategies should be applied depending on diagnosis and sample size.

\section{METHODOLOGICAL DESIGN}

Intervention design is another critical aspect. The assessment should be done by specialists, with specific training on the intervention program, and the evaluation process should be monitored.

Regarding duration, the intervention should be as intense as possible (e.g., daily and with a repeated design) to produce stronger effects relative to the preintervention baseline (Kanaan et al., 2014). In addition, the intervention should be long enough to produce changes in the assessed cognitive domains, but total duration should be reduced for small groups, which have not been controlled for individual differences). The longer the intervention, the higher the probability that uncontrolled individual/personal events will affect the results (Luttenberger et al., 2012).

Another important factor is specificity versus generality. Interventions focused on general aspects (quality of life and general cognitive status) are more methodologically demanding, because of the complexities of general skill training and measurement (Carrion et al., 2013). Such situations call for a more controlled, multi-measured, 
Table 1 | Relevant factors for improving research quality of cognitive interventions

\begin{tabular}{|c|c|}
\hline Participants & $\begin{array}{l}\text { Larger sample sizes allow for better control of confounding variables } \\
\text { The control and the intervention groups must be matched for critical clinical variables (dementia diagnosis, disease severity, } \\
\text { pharmacological treatment, caregiver support, etc.) }\end{array}$ \\
\hline $\begin{array}{l}\text { Methodological } \\
\text { design }\end{array}$ & $\begin{array}{l}\text { The assessment should be performed by specialists with specific training on the intervention } \\
\text { The intervention should be as intense as possible and total duration should be long enough to produce changes in the assessed } \\
\text { cognitive domains } \\
\text { When interventions are focused on general aspects, a more controlled, multi-measured, and multivariate design with a large sample } \\
\text { size should be considered } \\
\text { Application manuals and standardized protocols are required in order to maximize training suitability and protocol comparability }\end{array}$ \\
\hline $\begin{array}{l}\text { Control group } \\
\text { and intervention }\end{array}$ & $\begin{array}{l}\text { Both groups must be comparable regarding critical clinical variables that can affect cognition } \\
\text { Both groups should perform an activity that resembles the intervention in terms of intensity, duration, and social-physical } \\
\text { environments } \\
\text { Other confounding factors must be controlled for, such as adherence, incidental effects of external/personal events, and differences } \\
\text { in domains other than the content of intervention }\end{array}$ \\
\hline $\begin{array}{l}\text { Pre- and post- } \\
\text { measurements }\end{array}$ & $\begin{array}{l}\text { Different evaluators should be considered to assess pre/post measures as well as cognitive intervention } \\
\text { Measures targeting the specific cognitive domain trained should be used alongside more general measures } \\
\text { Both self-reports from patients and caregivers and objective measures (ranging from neuropsychology and experimental design to } \\
\text { brain function techniques, such as fMRI or EEG) must be considered }\end{array}$ \\
\hline $\begin{array}{l}\text { Statistical } \\
\text { concerns }\end{array}$ & $\begin{array}{l}\text { Statistical size effects are crucial in pre/post design } \\
\text { Multiple comparisons, multivariate designs, and corrections for multiple comparisons should be performed only with larger samples } \\
\text { For small samples, statistical assessment should be done at group-comparison level, with a focus on a single independent variable } \\
\text { indexing the intervention assessment } \\
\text { For small samples with a control group and pre/post assessment, analysis of multiple single cases should be a subsidiary strategy }\end{array}$ \\
\hline
\end{tabular}

and multivariate design (involving larger samples). Training in specific domains (e.g., working memory and emotional recognition) can be simpler for several reasons. There are relatively specific domains differentially affected in certain dementia subtypes [e.g., social cognition in bvFTD (Ibanez and Manes, 2012); memory in Alzheimer's disease (Parra et al., 2009)]. In these cases, targeted interventions may allow for more direct and intense training. Moreover, participant monitoring is more straightforward, pre/post measures can be directly related with the content of the intervention, and these effects can be more easily tracked with specific instruments.

Thus, intervention should be assessed by trained and controlled professionals, capable of assessing the trade-off between intensity, duration, and specificity versus generality in program design. A related problem is the heterogeneity of the interventions and their poor descriptions. Application manuals/handbooks and standardized protocols are required to maximize training efficiency and comparability of protocols (Hunsley and RumsteinMcKean, 1999; Town et al., 2012).

\section{CONTROL GROUP AND CONTROL VARIABLES}

The inclusion of a control group and control conditions increases the reliability and validity of the results. Repeated assessment and random aspects of personal experience have an impact on several cognitive domains. Thus, differences between pre- and post-measurements are not informative on their own. A control group should always be considered and, if possible, subjects must be randomly assigned to the experimental and control groups. The mock intervention or "placebo" effect usually emerges in studies involving unspecific social/recreational activities or no treatment at all (Bahar-Fuchs et al., 2013b). Control conditions should be carefully designed in order to hone specific intervention strategies.

Control group design should observe the following requisites: (a) patient characteristics should be measurable and comparable among groups; (b) the activities performed should be comparable with intervention tasks regarding intensity, duration, and social-physical environments; and (c) confounding factors should be controlled.
Regarding the latter consideration, a gold standard approach should monitor groups regarding adherence, incidental effects of external/personal events, and differences in domains other than the one being assessed. The patients and relatives' self-report scales and checklists prove very helpful for this strategy. If sample size allows, relevant individual differences should be measured and controlled by means of statistical comparisons. The patients' progression history, recent significant life events, and everyday behavior (diet, exercise, social, and recreational activities) should be controlled by means of group comparisons or multivariate analyses.

Therefore, the detection of intervention-specific effects requires a strict strategy including a truly comparable control group, a monitoring process, and, when possible, the control and weighing of confounding variables.

\section{PRE/POST MEASURES}

The difference between pre- and postintervention measures is perhaps the most important aspect to consider. A preintervention measure (or baseline) allows controlling for individual differences, and 
the effect of pre-post repetition can be disentangled by including a control group (Papp et al., 2009). Nevertheless, the most important issue is how to quantify the specific domain being trained.

One important factor is to favor the similarity between the measurements and the cognitive domain being tested. This is easier to address when a specific domain is being trained (e.g., working memory) because the similarity between the training and its measurement is restricted to an isolated aspect, and can be done by direct measurement of the task performance. Nevertheless, when general domains are considered (e.g., social functioning), the distance between the intervention and an isolated measure becomes evident (Owen et al., 2010), requiring complex or multiple measures (e.g., clinical variables, cognitive screening, life quality assessment, functionality measures, and relatives' reports). A more ambitious design should include specific and general training as well as specific and general pre/post-measurements. Note that a design as such requires a larger sample size, which is rarely observed in current reports.

Another important sub-aspect is the degree of objective measurement. Selfreports are adequate to assess patients' and relatives' subjective experiences of changes, but they lack objectivity and can be biased by different factors. Subjective reports should be accompanied by objective (implicit and explicit) measures, ranging from experimental designs to brain measures. Basic general cognitive assessments (e.g., MMSE and fluid intelligence) are usually considered in intervention programs, but their generality renders them insensitive to subtle changes.

Recent advances in translational cognitive neuroscience are promissory. Neuroimaging methods have demonstrated the sensitivity to training effects, suggesting their potential role as systematic measures of intervention success (Belleville et al., 2011). Electromagnetic techniques offer a simple, inexpensive approach to tap training effects (Vecchio et al., 2013; Yener and Basar, 2013). Moreover, recent breakthroughs in engineering have given rise to training-sensitive mathematical applications, such as the case of brain connectivity measures (with both neuroimaging and electromagnetic techniques) (Pievani et al., 2011).

In brief, an adequate integrated approach to assess cognitive intervention effects requires the use of multi-level measures (self-reports, neuropsychology, and neuroscience) and a balance between specificity and generality.

\section{STATISTICAL ISSUES}

Finally, statistical soundness is crucial to attain reliability, validity, and replicability. Although sample size and the assessment of confounding variables drive most of the considerations, there are important decisions, which can bias the results. First of all, statistical size effects are more imperative in pre/post design than in basic research. Thus, they should be always reported, because they offer direct information about the power of the intervention.

Multiple comparisons and multivariate designs should be considered only with larger samples; otherwise, the statistical validity of the results will prove highly questionable. Corrections for multiple comparisons and selection of complex statistical designs (e.g., structural equation models, discriminant analysis, or support vector machine) should also be restricted to larger samples. Multi-site studies represent a current solution to sample size problems.

For small samples, statistical assessment should be done at group-comparison level. The focus should be on a single independent variable indexing the intervention assessment. A valid alternative in the context of small samples with a control group and pre/post assessment would be the analysis of multiple single cases (McIntosh and Brooks, 2011; Sajjadi et al., 2013), allowing a deeper examination of intervention effects.

\section{CONCLUSION}

Much more knowledge is required to understand the dramatic brain changes triggered by dementia and the parallel effects of training during cognitive intervention. The assessment of patients with dementia tends to underestimate several methodological considerations, sometimes yielding inconsistent, unreliable, or weak results. Here, we have highlighted important decisions, which may impact positively on research quality (see Table $\mathbf{1}$ for a summary of these issues). An enhanced control and systematization of these issues will improve our understanding of how cognitive training affects the patients' and relatives' quality of life.

\section{ACKNOWLEDGMENTS}

This work was partially supported by grants from CONICET, CONICYT/FONDECYT Regular (1130920), FONCyT-PICT 2012-0412, FONCyTPICT 2012-1309, and the INECO Foundation.

\section{REFERENCES}

Bahar-Fuchs, A., Clare, L., and Woods, B. (2013a). Cognitive training and cognitive rehabilitation for mild to moderate Alzheimer's disease and vascular dementia. Cochrane Database Syst. Rev. 6, CD003260. doi:10.1002/14651858.CD003260. pub2

Bahar-Fuchs, A., Clare, L., and Woods, B. (2013b). Cognitive training and cognitive rehabilitation for persons with mild to moderate dementia of the Alzheimer's or vascular type: a review. Alzheimers Res. Ther. 5, 35. doi:10.1186/alzrt189

Belleville, S., Clément, F., Mellah, S., Gilbert, B., Fontaine, F., and Gauthier, S. (2011). Trainingrelated brain plasticity in subjects at risk of developing Alzheimer's disease. Brain 134(Pt 6), 1623-1634. doi:10.1093/brain/awr037

Carrion, C., Aymerich, M., Baillés, E., and LópezBermejo, A. (2013). Cognitive psychosocial intervention in dementia: a systematic review. Dement. Geriatr. Cogn. Disord. 36, 363-375. doi:10.1159/ 000354365

Dröes, R. M., van der Roest, H. G., van Mierlo, L., and Meiland, F. J. (2011). Memory problems in dementia: adaptation and coping strategies and psychosocial treatments. Expert Rev. Neurother. 11, 1769-1781. doi:10.1586/ern.11.167

Fernández-Prado, S., Conlon, S., Mayán-Santos, J. M., and Gandoy-Crego, M. (2012). The influence of a cognitive stimulation program on the quality of life perception among the elderly. Arch. Gerontol. Geriatr. 54, 181-184. doi:10.1016/j.archger.2011. 03.003

Giordano, M., Dominguez, L. J., Vitrano, T., Curatolo, M., Ferlisi, A., Di Prima, A., et al. (2010). Combination of intensive cognitive rehabilitation and donepezil therapy in Alzheimer's disease (AD). Arch. Gerontol. Geriatr. 51, 245-249. doi:10.1016/j. archger.2009.11.008

Hunsley, J., and Rumstein-McKean, O. (1999). Improving psychotherapeutic services via randomized clinical trials, treatment manuals, and component analysis designs. J. Clin. Psychol. 55, 1507-1517. doi:10.1002/(SICI)1097-4679(199912) 55:12<1507::AID-JCLP8>3.0.CO;2-A

Hurd, M. D., Martorell, P., Delavande, A., Mullen, K. J., and Langa, K. M. (2013). Monetary costs of dementia in the United States. N. Engl. J. Med. 368, 1326-1334. doi:10.1056/NEJMsa1204629

Ibanez, A., and Manes, F. (2012). Contextual social cognition and the behavioral variant of frontotemporal 
dementia. Neurology 78, 1354-1362. doi:10.1212/ WNL.0b013e3182518375

Kanaan, S. F., McDowd, J. M., Colgrove, Y., Burns, J. M., Gajewski, B., and Pohl, P. S. (2014). Feasibility and efficacy of intensive cognitive training in early-stage Alzheimer's disease. Am. J. Alzheimers Dis. Other Demen. 29, 150-158. doi:10. 1177/1533317513506775

Luttenberger, K., Hofner, B., and Graessel, E. (2012). Are the effects of a non-drug multimodal activation therapy of dementia sustainable? Follow-up study 10 months after completion of a randomised controlled trial. BMC Neurol. 12:151. doi:10.1186/ 1471-2377-12-151

McIntosh, R. D., and Brooks, J. L. (2011). Current tests and trends in single-case neuropsychology. Cortex 47, 1151-1159. doi:10.1016/j.cortex.2011.08.005

Niu, Y. X., Tan, J. P., Guan, J. Q., Zhang, Z. Q., and Wang, L. N. (2010). Cognitive stimulation therapy in the treatment of neuropsychiatric symptoms in Alzheimer's disease: a randomized controlled trial. Clin. Rehabil. 24, 1102-1111. doi:10. 1177/0269215510376004

Olazarán, J., Reisberg, B., Clare, L., Cruz, I., PeñaCasanova, J., Del Ser, T., et al. (2010). Nonpharmacological therapies in Alzheimer's disease: a systematic review of efficacy. Dement. Geriatr. Cogn. Disord. 30, 161-178. doi:10.1159/000316119

Owen, A. M., Hampshire, A., Grahn, J. A., Stenton, R., Dajani, S., Burns, A. S., et al. (2010). Putting brain training to the test. Nature 465, 775-778. doi:10.1038/nature 09042

Papp, K. V., Walsh, S. J., and Snyder, P. J. (2009). Immediate and delayed effects of cognitive interventions in healthy elderly: a review of current literature and future directions. Alzheimers Dement. 5, 50-60. doi:10.1016/j.jalz.2008.10.008
Parra, M. A., Abrahams, S., Fabi, K., Logie, R., Luzzi, S., and Della Sala, S. (2009). Short-term memory binding deficits in Alzheimer's disease. Brain 132(Pt 4), 1057-1066. doi:10.1093/brain/awp036

Pievani, M., de Haan, W., Wu, T., Seeley, W. W., and Frisoni, G. B. (2011). Functional network disruption in the degenerative dementias. Lancet Neurol. 10, 829-843. doi:10.1016/S1474-4422(11)70158-2

Prince, M., Bryce, R., and Ferr, C. (2011). World Alzheimer Report 2011. London, UK: Alzheimer's Disease International, 69.

Sajjadi, S. A., Acosta-Cabronero, J., Patterson, K., Diazde-Grenu, L. Z., Williams, G. B., and Nestor, P. J. (2013). Diffusion tensor magnetic resonance imaging for single subject diagnosis in neurodegenerative diseases. Brain 136(Pt 7), 2253-2261. doi:10.1093/brain/awt118

Town, J. M., Diener, M. J., Abbass, A., Leichsenring, F., Driessen, E., and Rabung, S. (2012). A metaanalysis of psychodynamic psychotherapy outcomes: evaluating the effects of research-specific procedures. Psychotherapy (Chic) 49, 276-290. doi: 10.1037/a0029564

Vecchio, F., Babiloni, C., Lizio, R., Fallani, F. V., Blinowska, K., Verrienti, G., et al. (2013). Resting state cortical EEG rhythms in Alzheimer's disease: toward EEG markers for clinical applications: a review. Suppl. Clin. Neurophysiol. 62, 223-236. doi:10.1016/B978-0-7020-5307-8.00015-6

Viola, L. F., Nunes, P. V., Yassuda, M. S., Aprahamian, I., Santos, F. S., Santos, G. D., et al. (2011). Effects of a multidisciplinary cognitive rehabilitation program for patients with mild Alzheimer's disease. Clinics (Sao Paulo) 66, 1395-1400. doi:10.1590/S180759322011000800015

Wollen, K. A. (2010). Alzheimer's disease: the pros and cons of pharmaceutical, nutritional, botanical, and stimulatory therapies, with a discussion of treatment strategies from the perspective of patients and practitioners. Altern. Med. Rev. 15, 223-244.

Woods, B., Aguirre, E., Spector, A. E., and Orrell, M. (2012). Cognitive stimulation to improve cognitive functioning in people with dementia. Cochrane Database Syst. Rev. 2, CD005562. doi:10.1002/ 14651858.CD005562.pub2

Yener, G. G., and Basar, E. (2013). Biomarkers in Alzheimer's disease with a special emphasis on event-related oscillatory responses. Suppl. Clin. Neurophysiol. 62, 237-273. doi:10.1016/B978-07020-5307-8.00020-X

Conflict of Interest Statement: The authors declare that the research was conducted in the absence of any commercial or financial relationships that could be construed as a potential conflict of interest.

Received: 16 May 2014; accepted: 01 August 2014; published online: 13 August 2014.

Citation: Ibanez A, Richly P, Roca $M$ and Manes F (2014) Methodological considerations regarding cognitive interventions in dementia. Front. Aging Neurosci. 6:212. doi: 10.3389/fnagi.2014.00212

This article was submitted to the journal Frontiers in Aging Neuroscience.

Copyright $\odot 2014$ Ibanez, Richly, Roca and Manes. This is an open-access article distributed under the terms of the Creative Commons Attribution License (CC BY). The use, distribution or reproduction in other forums is permitted, provided the original author(s) or licensor are credited and that the original publication in this journal is cited, in accordance with accepted academic practice. No use, distribution or reproduction is permitted which does not comply with these terms. 